\title{
Besondere Sitzung vom 31. Oktober 1908.
}

Vorsitzender: Hr. W. Nernst, Präsident.

Der Präsident begrüßt die in der außerordentlich zahlreichen Versammlung anwesenden Gäste aus medizinischen Kreisen, besonders Se. Exzellenz Hrn. Wirkl. Geh. Med.-Rat Robert Koch, sowie die Vertreter des Reichsgesundbeitsamts, des Instituts für Infektionskrankheiten, der Physiologischen und Pharmazeutischen Gesellschaft.

Sodann heißt er die auswärtigen Mitglieder, Hrn. Dr. L. Ach (Mannheim) und Geh. Rat Prof. Dr. L. Knorr (Jena) und besonders den Redner der Sitzung, Hrn. Geh. Ober-Medizin.-Rat Prof. Dr. P. Ehrlich (Frankfurt a. M.) willkommen und erteilt letzterem das Wort zu seinem zusammenfassenden Vortrage:

"Über den jetzigen Stand der Chemotherapie."

Nach Beendigung des Vortrages, der mit lebhaftestem Beifall aufgenommen wurde, schließt der Vorsitzende die Sitzung mit der folgenden Ansprache:

\section{"Hochverehrte Anwesende!}

Der Versammlung, die unserem Ehrengaste bereits so lebhaft den Dank zum Ausdruck gebracht hat, möchte ich mich noch mit einigen Worten anschließen. Das Gebiet, das Sie, bochverehrter Herr Kollege, seit einer Reihe von Jahren mit so viel Erfolg bearbeiten, galt vielfach als dem Naturforscher unnahbar. Wenn man sogar Bedenken trug, aul die kosmischen Probleme die Prinzipien der Physik und Chemie bis auf ihre letzten Konsequenzen anzuwenden, so erscheint es erst recht gewagt, die Theorie der lebenden Zelle auf den gleichen Prinzipien aufzubauen. Sowohl in Ihren früheren Arbeiten wie in Ihrem heutigen Vortrage glaube ich überall den gleichen Grundton herauszuhören, nämlich die Übertragung der logischen und experimentellen Methoden der organischen Chemie mit allen ibren Konsequenzen auch auf die lebende Zelle. Sie haben gezeigt, daß man die Zelle in vieler Hinsicht theoretisch wie ein Berichte d. D. Chem. Gesellschaft Jahrg. XXXXI. 
Molekül einer organischen Verbindung behandeln kann; allerdings lesitzt die Zelle so zahlreiche Atomgruppen und entsprechende Affinitätsäußerungen, daß man mit ihrer Beschreibung wohl einen neuen "Beilstein«| füllen könnte. Aber einzelne Valenzäußerungen haben sich klar aus der Kompliziertheit der Erscheinungen herausschälen lassen, und so haben Sie erreicht, daß Ihre Arbeiten nicht nur dem Erkenntnistrieb auf einem der bedeutsamsten Gebiete entgegenkommen, sondern auch der praktischen Therapie so manchen wichtigen Fingerzeig liefern.

Ein viel zitiertes Wort sagt: »die edelste Beschäftigung des Menschen ist der Mensch." Wir Naturforscher werden dem wohl nicht ganz zustimmen können, aber unter dem Eindruck Ihres Vortrages werden wir zu konzedieren bereit sein, daß die edelste Beschäftigung des Naturforschers die lebende Zelle sei. Und so möchte ich mit dem nochmaligen Danke den Glückwunsch vereinen, daß Sie ein so schönes Arbeitsfeld sich erwählt haben.«
Der Vorsitzende:
Der Schriftführer:
W. Nernst.
C. Schotten.

\title{
Sitzung vom 9. November 1908.
}

\author{
Vorsitzender: Hr. W. Nernst, Präsident.
}

\section{Das Protokoll der letzten Sitzung wird genehmigt.}

Der Vorsitzende begrüßt das auswärtige Mitglied, Hrn. Prof. Dr. B. Lepsius, Griesheim, und macht alsdann folgende Mitteilung:

»Hr. Dr. P. W. Hofmann, Darmstadt, hat eine Anzahl Briefe, die sein Onkel A. W. v. Hofmann an ihn gerichtet hat, unserer Gesellschaft übergeben. Wenn diese Briefe auch nicht wissenschaftlichen Inhalt haben, so legen sie doch Zeugnis ab von der großen Herzensgüte des Mannes, den wir als unseren ersten Präsidenten verehren. Die Sammlung ist in einem prächtigen Einband enthalten und hier vor Ihren Augen aufgestellt; sie wird fortan als Erinnerung an A. W. v. Hofmann im Hofmannhause aufbewabrt werden.

Dem Stifter dieser Sammlung sprechen wir unseren wärmsten Dank aus." 